\title{
Curcumin solid dispersion based on three model acrylic polymers: formulation and release properties
}

\author{
Shuai Zong1,2, Yuting Liu ${ }^{1}$, Hyun Jin Park³ ${ }^{3}$ Ming Ye ${ }^{1}$, Jinglei Li ${ }^{\oplus *}$ \\ ${ }^{I}$ School of Food and Biological Engineering, Hefei University of Technology, \\ Hefei, PR China, ${ }^{2}$ College of Food Science and Engineering, Yangzhou University, \\ Yangzhou 225127, Jiangsu, PR China, ${ }^{3}$ School of Life Sciences and Biotechnology, \\ Korea University, 5-Ka, Anam-Dong, Sungbuk-Ku, Republic of Korea \\ First author: Shuai Zong
}

\begin{abstract}
To investigate structure-property relationship of polymer-based curcumin solid dispersion (SD), three acrylic polymers were used to formulate curcumin SD by solvent evaporation method. Curcumin Eudragit EPO SD (cur@EPO), curcumin Eudragit RS PO SD (cur@RSPO) and curcumin Eudragit RL PO SD (cur@RLPO) showed deep red, golden orange and reddish orange color, respectively. Cur@RSPO entrapped 15.42 wt $\%$ of curcumin followed by cur@RL PO and cur@EPO. FTIR spectra indicated that in cur@EPO, curcumin may transfer hydrogen to the dimethylaminoethyl methacrylate group and thus change its color to red. In contrast, curcumin may form hydrogen bonding with Eudragit RS PO and Eudragit RL. Curcumin exists in amorphous state in three SDs as proved by differential scanning calorimetry and X-Ray diffraction measurement. In vitro digestion presented that lower $\mathrm{pH}$ value in simulated gastric fluid (SGF) stimulates the curcumin release from cur@EPO while permeability influences the release profile in other two SDs. When in simulated intestinal fluid (SIF), first order release model governs the release behaviors of all three SDs which showed sustained release pattern. Our results are helpful to elucidate how structure of polymer may impact on the major properties of curcumin contained SD and will be promising to broaden its therapeutic applications.
\end{abstract}

Keywords: Control release. Curcumin. Solid dispersion. In vitro digestion. Eudragit polymer.

Chemical compounds studied in this article

Curcumin (PubChem CID: 969516); Eudragit E PO (PubChem CID: 107676); Eudragit RS PO

(PubChem CID: 104931); Eudragit RL PO (PubChem CID: 104804)

\section{INTRODUCTION}

Curcumin [(1E, 6E)-1, 7-bis (4-hydroxy-3-methoxyphenyl)-1, 6-heptadiene-3, 5-ione] is the primary active compound of the perennial herb Curcuma Longa L. (turmeric) which has been used as nutritional supplement and herbal medicine in many Asian countries for thousands of years (Kharat, Zhang, McClements, 2018). In recent decades, curcumin has been extensively investigated for its broad spectrum of therapeutic properties, such as anti-oxidant, anti-inflammatory, anti-cancer, anti-

*Correspondence: J. Li. School of Food and Biological Engineering. Hefei University of Technology. Hefei, Anhui 230009, P.R. China. Phone: +860551-62919368. Fax: +86-0551-62911368. E-mail: lijinglei2012@hotmail. com microbial, wound healing, and potential prevention ability of neurodegenerative diseases (Boyanapalli et al., 2018; Deck et al., 2018; Soleimani et al., 2018). Examined by thousands of years' usage and several clinical trials, curcumin has very good safety profile: as high as $8 \mathrm{~g}$ /day dosage did not cause any adverse effects (Sanmukhani, Anovadiya, Tripathi, 2011).

However, curcumin has extremely low solubility and dissolution rate in aqueous media due to strong inter and intra-molecular hydrogen bonds (Heger et al., 2014). It has slightly higher solubility in alkaline solution but is degraded quickly into vanillin, ferulic acid, and feruloyl methane (Tønnesen, Karlsen, 1985). To circumvent the inherent limitations and promote the applications of curcumin in the pharmaceutical industry, 
several methods, including nano-formulation, physical inclusion and chemical modification, have been proposed and investigated (Li et al., 2015a, b; Tang et al., 2002). But most of the preparation methods are very complicated and require large amount of organic solvent which is problematic and costly in the practical applications.

Recently, polymer based amorphous solid dispersion (SD) was also reported to overcome the problems associated with curcumin (Paradkar et al., 2004; Zhang et al., 2011). Not like aforementioned approaches, SD is relatively easy to prepare, with higher loading ability and stability. It is also scalable and economic which makes this method particular appealing in the pharmaceutical industry. In our previous study, curcumin Eudragit E PO (cur@EPO) SD was fabricated using solution mixing method ( $\mathrm{Li}$ et al., 2015a). The formulated SD greatly increased the water solubility, stability as well as bioavailability of curcumin. However, in our previous study the prepared SD contained significant amount of sodium acetate which was introduced from the buffer solution. Moreover, the interaction between curcumin and the dispersing polymer was not clearly elucidated. It was suggested that the positive charge of the polymer may contribute to the hydrogen bond formation with curcumin but the proof is inadequate.

In the present study, we prepared curcumin SD using the solvent evaporation method along with three model acrylic polymers: Eudragit E PO, Eudragit RS PO and Eudragit RL PO. Eudragit E PO is a polyelectrolyte that belongs to the family of (meth) acrylate copolymers. It is composed of dimethylaminoethyl methacrylate, butyl methacrylate, and methyl methacrylate with the molar ratio of 2:1:1 (Gallardo, Skalsky, Kleinebudde, 2008). Eudragit E PO is generally regarded as nontoxic, nonirritant and essentially safe, which is widely used in the pharmaceutical industry for various purposes such as taste masking, moisture protection, a film coating, modified release (Gallardo, Skalsky, Kleinebudde, 2008). Eudragit RL PO and Eudragit RS PO are similar copolymers based on ethyl acrylate, methyl methacrylate and a low content of a methacrylic acid ester with quaternary ammonium groups. The molar ratio of ethyl acrylate, methyl methacrylate and trimethylammonioethyl methacrylate is approximately
1:2:0.2 for Eudragit RL PO and approximately 1:2:0.1 for Eudragit RS PO. Both Eudragit RL PO and Eudragit RS PO are insoluble in aqueous solution and used widely as release control agents (Kim et al., 2002). The three acrylic polymers are mainly used in the formulation of dosage forms, most commonly in the preparation of matrix tablets for oral sustained release and in tablet coating (Crotts et al., 2001; Takka, Rajbhandari, Sakr, 2001). Few studies have been carried out to exploit their potential in the amorphous drug formulation. Eudragit E PO contains dimethylaminoethyl methacrylate section, while Eudragit RS PO and Eudragit RL PO contain different amount of trimethylammonioethyl methacrylate. It is hypothesized that these three Eudragit polymers could form SD with curcumin, enhance its solubility and regulate its release pattern. But Eudragit polymers have different chemical structure which will exert impact on their ability to form curcumin SD and pertaining physicochemical properties. Herein, it is aimed in this study to prepare curcumin SDs with three model Eudragit polymers and compare their major properties via DSC, XRD, and FTIR measurements. The release of curcumin from the SDs in simulated gastrointestinal fluid were also conducted and compared. The results of this study can provide an insight into acrylic polymer based SD formulation which is helpful to promote the broad therapeutic activities of curcumin.

\section{MATERIAL AND METHODS}

\section{Material}

Curcumin with 98\% purity was purchased from Acros Organics (New Jersey, USA). Eudragit E PO, Eudragit RS PO and Eudragit RL PO were provided by Evonik Industries AG (Darmstadt, Germany) and used as received. Syringe filter, $0.45 \mu \mathrm{m}$, was obtained from Pall life science (Ann Arbor, MI, USA). All other reagents and solvents were of analytical grade and used as received.

\section{Preparation procedure}

Solvent evaporation method was utilized to develop curcumin SD (Changdeo et al., 2011). Polymer was 
dispersed in acetone at the concentration of $10 \mathrm{mg} / \mathrm{mL}$ in a glass baker. After the polymer was fully dissolved in the solvent, curcumin acetion solution was added by a syringe at $20 \%$ of the weight of polymer. The mixture was further stirred by a magnetic stirring for $5 \mathrm{~h}$ before passed through syringe filter $(0.45 \mu \mathrm{m})$ to remove possible curcumin crystal particles. Acetone was completely removed by vacuum rotary evaporation for about $1 \mathrm{~h}$. Curcumin and polymer physical mixture was prepared by mixing curcumin with corresponding polymer in the same weight ratio with a mortar and pestle.

\section{Loading ability}

Loading ability was defined as the weight ratio of curcumin to corresponding polymer. The content of curcumin was calculated according to a calibration formula listed below:

$\mathrm{Y}=0.1734 * \mathrm{X}-0.0207, \mathrm{r}^{2}=0.9994$

where $\mathrm{Y}$ was the absorption at $419 \mathrm{~nm}$ and $\mathrm{X}$ was the concentration of curcumin $(\mu \mathrm{g} / \mathrm{mL})$.

The calibration curve was calculated using the absorption readings at $419 \mathrm{~nm}$ of several standard curcumin acetone solutions (within the concentration range of 0-10 $\mu \mathrm{g} / \mathrm{mL}$ ). SD samples were dissolved in acetone and the absorption readings at $419 \mathrm{~nm}$ were recorded.

\section{Differential scanning calorimetry (DSC)}

Thermal transition properties of curcumin, Eudragit polymers, curcumin and polymer physical mixture, and curcumin polymer SD were conducted on a model Q20 differential scanning calorimeter (TA Instrument, New Castle, USA). Sample powder (about $5.0 \mathrm{mg}$ ) was weighted and sealed in an aluminum DSC pan. After holding isothermally at $50{ }^{\circ} \mathrm{C}$ for $1 \mathrm{~min}$, DSC scanning was performed from $50{ }^{\circ} \mathrm{C}$ to $200{ }^{\circ} \mathrm{C}$ at a heating rate of $10{ }^{\circ} \mathrm{C} / \mathrm{min}$ under dry nitrogen purge of $50 \mathrm{~mL} / \mathrm{min}$. An empty pan was served as reference.

\section{X-Ray diffraction (XRD)}

Crystalline phase was identified through XRD analysis. The measurement was conducted on an X-ray diffractometer (X'Pert PW3040/00, Philips, Almelo, Netherlands) equipped with $\mathrm{Cu}-\mathrm{K} \alpha$-radiation with K-A2/ $\mathrm{K}-\mathrm{A} 1$ ratio of 0.5 , generated at $30 \mathrm{~mA}$ and $40 \mathrm{kV}$. The scanning was operated in the range between $5-40^{\circ} 2 \theta$ with a step-angle of $0.02^{\circ}$ at a scan rate of $1 \% \mathrm{~min}$.

\section{Attenuated total reflecting-Fourier-transform infrared (ATR-FTIR) investigation}

The possible interaction between curcumin and each polymer was investigated by ATR-FTIR. Curcumin SDs were carefully grinded by the mortar and pestle to break down large particles. Spectra of samples were acquired on a Perkin-Elmer Spectrum 100 spectrophotometer (Waltham, MA, USA) in the wavelength region between 600 and $4000 \mathrm{~cm}^{-1}$. Each spectrum was obtained by averaging 32 scans at a resolution of $4 \mathrm{~cm}^{-1}$.

\section{Curcumin release profile in simulated digestion fluid}

Release profile of curcumin in simulated gastric fluid (SGF) and simulated intestinal fluid (SIF) was examined in accordance with previous publications with certain modifications (Esmaili et al., 2011; Paramera, Konteles, Karathanos, 2011). SGF was prepared by dissolving $2.0 \mathrm{~g}$ of $\mathrm{NaCl}, 7.0 \mathrm{~mL}$ of $\mathrm{HCl}(36 \mathrm{w} / \mathrm{w} \%)$ and $1 \mathrm{~g}$ of Tween 80 into 1 liter of distilled water, $\mathrm{pH}$ value was adjusted to 1.2. For SIF, $6.8 \mathrm{~g}$ of $\mathrm{KH}_{2} \mathrm{PO}_{4}, 0.616 \mathrm{~g}$ of $\mathrm{NaOH}$ and $1 \mathrm{~g}$ of Tween 80 was dissolved in 1 liter of distilled water, $\mathrm{pH}$ was adjusted to 6.8 . About $5 \mathrm{mg}$ of SD or physical mixture was weighted to a glass vial to which $50 \mathrm{~mL}$ of pre-warmed SIF or SGF was added. Pure curcumin (about $1 \mathrm{mg}$ ) was used as control. Glass vials were transferred to a water bath set at $37{ }^{\circ} \mathrm{C}$ and $120 \mathrm{rpm}$ of shaking for the release measurement. Release of curcumin was monitored on a UV-vis spectrometer at $419 \mathrm{~nm}$.

To further understand release kinetics of curcumin from various formulations, the release profile was fitted with four experiential kinetic models: zero-order, Higuchi, first-order and Ritger-peppas release model (Desai, Simonelli, Higuchi, 1965; Higuchi, 1963; Korsmeyer et al., 1983; Najib, Suleiman, 1985). 
For the zero-order equation: $\mathrm{Q}=\mathrm{kt}$; for Higuchi equation: $\mathrm{Q}=\mathrm{kt}^{1 / 2}$; for first-order release equation: $\ln (1-\mathrm{Q})=-\mathrm{kt}$; and for Ritger-peppas release: $\mathrm{Q}=\mathrm{kt}^{\mathrm{n}}$. In these equations, $Q$ is release percentage of drug at time point $\mathrm{t}$, while $\mathrm{k}$ is the kinetic constant. In the Ritgerpeppas equation, $\mathrm{n}$ is the diffusional exponent.

\section{RESULTS AND DISCUSSION}

\section{Sample preparation and loading ability}

Polymer solid dispersion (SD) is an effective strategy to deliver lipophilic drugs that have low solubility and bioavailability. Methods like coextrusion, spray-drying, freeze-drying and film casting are extensively investigated for the formulation (Breitenbach, 2002). In the present study, solvent evaporation method was applied to manufacture SD which contains only curcumin and corresponding polymer. It is notable to point out that curcumin Eudragit E PO SD (cur@EPO) has deep red color while other two SDs (cur@RSPO and cur@RLPO) show curcumin characteristic yellow color (Figure S1). When dissolved in acetone, the color difference of respective SD was not significant in solution state but became remarkable during the evaporation of solvent. UV absorption survey of cur@EPO, cur@RSPO and cur@RLPO acetone solution exhibited similar absorption value as well as profile compared with that of pure curcumin acetone solution, implying that after dispersed in the polymer no chemical structure changes happened (Figure S2). Loading ability of each SD was listed in Table I. Cur@ RSPO showed higher loading ability for curcumin than both cur@EPO and cur@RLPO. The loading ability result is consistent with our previous report that Eudragit RS PO formulated microspheres also encapsulated higher level of lipophilic drug than that of Eudragit RL PO (Kim et al., 2002). Molecular structure as well as the ratio of each copolymer unit may account for the observed difference. Eudragit RS PO and RL PO only differs in the number of trimethylammonioethyl methacrylate in the way that RS PO contains about $50 \%$ less of this unit than that of RL PO. It is also reported that RS PO is slightly more hydrophobic than RL PO which may also contribute to its higher loading ability (Sahoo et al., 2009). During the preparation, curcumin was fully mixed with Eudragit RS PO in amorphous state. In the microenvironment, curcumin may interact with functional groups like carbonyl or amine group, thus increase its loading ability. The higher level of trimethylammonioethyl methacrylate in RL PO may decrease the affinity between the corresponding polymer and curcumin thus reducing its loading amount. 
<smiles>COC(=O)C(C)(CC(C)(C)C(=O)OCCN(C)C)CC(C)(C(=O)OC)C(C)(C)C</smiles>

Eudragit E PO<smiles>[R20][Z11](CC)(CC)CCOC(=O)C(C)(C)CC(C)(CC(C)(C)C(=O)OC)C(=O)OC</smiles><smiles>CCOC(=O)C(C)(C)CC(C)(CC(C)(C)C(=O)OC)C(=O)OC</smiles>

Eudragit RS PO<smiles>C[N+](C)(C)[Cl+3]</smiles><smiles>COc1cc(/C=C/C(=O)CC(=O)/C=C/c2ccc(O)c(OC)c2)ccc1O</smiles>

Curcumin

FIGURE 1 - Chemical structure of Eudragit E PO, Eudragit RS PO, Eudragit RL PO and curcumin.

TABLE I - Loading ability of curcumin solid dispersion and their appearance

\begin{tabular}{lcc}
\hline Solid dispersion sample $^{\mathbf{a}}$ & Loading ability (\%) $^{\mathbf{b}}$ & Appearance \\
\hline Cur@EPO & $11.66 \pm 0.06$ & Deep red color powder \\
\hline Cur@RSPO & $15.42 \pm 0.10$ & Golden orange \\
\hline Cur@RLPO & $12.86 \pm 0.09$ & Reddish orange \\
\hline
\end{tabular}

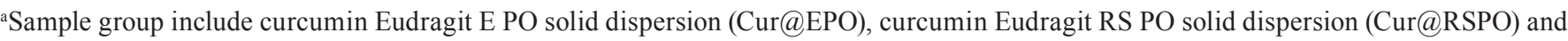
curcumin Eudragit RL PO solid dispersion (Cur@RLPO).

${ }^{b}$ Loading ability was calculated by the weight of curcumin and polymer and expressed as mean $\pm \mathrm{SD}, \mathrm{n}=3$.

\section{Differential scanning calorimetry (DSC) and $X$-Ray diffraction (XRD) investigation}

As pointed in previous researches, curcumin is a moderate hydrophobic phenolic compound whose partition-coefficient is about $\log \mathrm{P} 2.5$, but possesses strong inter and intra-molecular hydrogen bond, rendering crystal curcumin hard to be dissolved in aqueous medium
(Hasan et al., 2014). Polymer based SD is an effective method to change curcumin from crystal structure to amorphous state, conferring higher dissolution rate as well as bio-availability.

DSC was used to probe the melting and crystallinity profile of pristine curcumin powder, Eudragit polymers, curcumin and Eudragit polymer physical mixture, and curcumin SD samples (Figure 2). The DSC curve of 
pristine curcumin showed a single endothermic peak at $178.8^{\circ} \mathrm{C}$ indicating the crystal melting point (Kakran et al., 2012). DSC thermogram curves of cur@EPO, cur@ RSPO and cur@RLPO exhibited no obvious endothermic peak at around the melting point of curcumin, indicating that curcumin may loss its crystallinity in the matrix of Eudragit polymers. Curcumin may exist in amorphous state in the SD which is in line with our previous study (Li et al., 2015a). Curcumin, when exist in amorphous state, is not stable and tend to recrystallization at elevated temperature (Pawar et al., 2012). Our result suggest that curcumin in SD maintained the amorphous state after rotary.

In order to further confirm the change of physical state of curcumin after entrapped into matrix of Eudragit polymers, XRD was utilized to detect the crystallization properties (Figure 3). XRD pattern of Eudragit polymers exhibited no significant diffraction peaks which is in line with the amorphous nature of these three acrylic polymers as previously reported (Liu et al., 2010; Varshosaz, Faghihian, Rastgoo, 2006; Wu, McGinity, 2003). XRD pattern of pure curcumin demonstrated diffraction peaks at $8.98^{\circ}, 12.26^{\circ}, 14.62^{\circ}, 15.22^{\circ}, 17.42^{\circ}, 18.26^{\circ}$, and $22.82^{\circ} 2 \theta$. On the other hand, all the three curcumin SD samples showed an amorphous character without defined peaks which is similar to that of corresponding Eudragit polymer. The halo XRD pattern of curcumin SDs implies that no crystal compound exist, thus reinforces the observation of DSC that in the all three acrylic polymer based SDs, curcumin exists in amorphous state rather than crystal state.
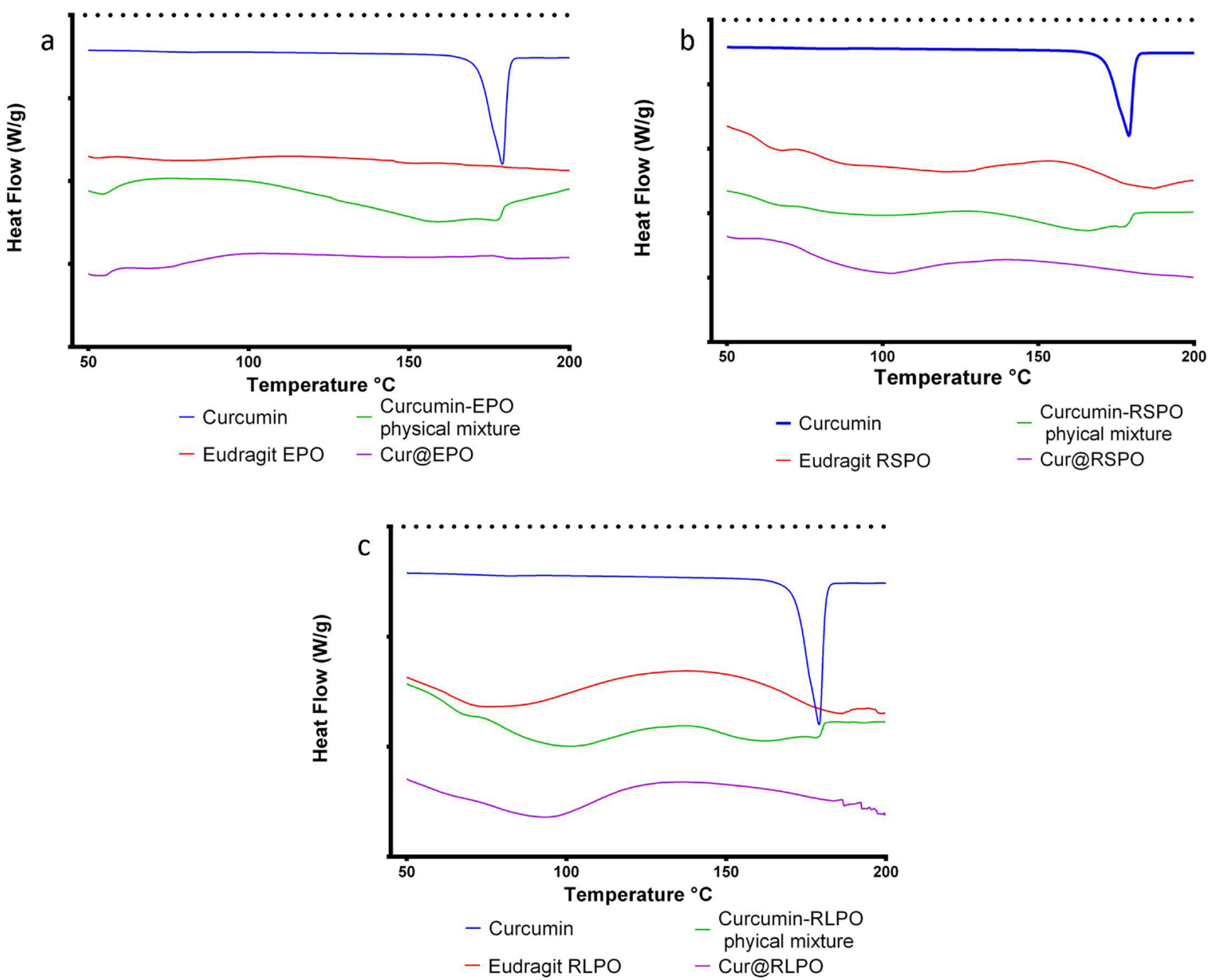

FIGURE 2 - Differential scanning calorimetry (DSC) thermograms of a: curcumin, Eudragit E PO, physical mixture and curcumin Eudragit E PO solid dispersion; b: curcumin, Eudragit RS PO, physical mixture and curcumin Eudragit RS PO solid dispersion; c: curcumin, Eudragit RL PO, physical mixture and curcumin Eudragit RL PO solid dispersion. 

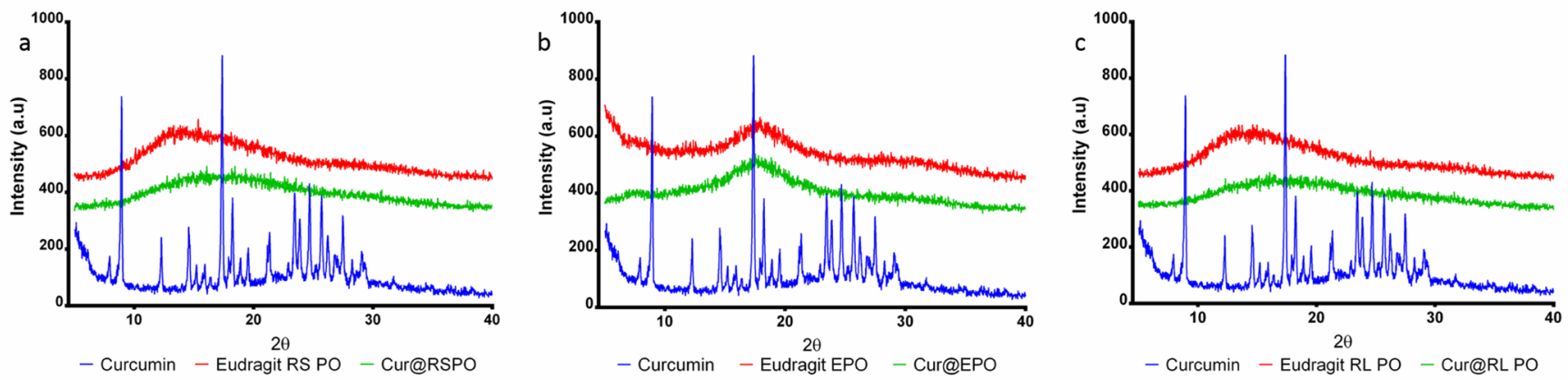

FIGURE 3 - X-Ray diffraction (XRD) profiles of a: curcumin, Eudragit RS PO and curcumin Eudragit RS PO solid dispersion; b: curcumin, Eudragit E PO and curcumin Eudragit E PO solid dispersion; c: curcumin, Eudragit RL PO and curcumin Eudragit RL PO solid dispersion.

\section{Attenuated total reflecting-Fourier-transform infrared (ATR-FTIR) investigation}

The possible interaction between curcumin and polymer was studied through FT-IR analysis. Even though curcumin possesses extremely low solubility in aqueous media, with the help of some polymers, curcumin could be well dissolved in water (Wegiel et al., 2014). However, the interaction between curcumin and the polymers were not well elucidated. In our previous study, hydrogen bond between curcumin and Eudragit E PO was determined as the major interaction (Li et al., 2015a). Nevertheless, it is still not clear how the molecular structure of SD polymer may impact on the interaction and other relevant properties. Polymorphism of curcumin is reported in several articles. The solution and other environmental factors like temperature, $\mathrm{pH}$ value may interfere with the crystalline formation procedure (Thorat, Dalvi, 2015; Sanphui et al., 2011). But in the present study, curcumin exist in amorphous state, as proved by XRD and DSC result. The microenvironment created by different $\mathrm{SD}$ polymer may impact on the interaction of curcumin and polymer, which need further studies.

In Figure 4, FT-IR spectra of Eudragit E PO, RSPO, RLPO, curcumin and corresponding polymer physical mixture and three SD samples were depicted. As displayed in Figure 4a, physical mixture of curcumin with Eudragit E PO exhibited both the characteristic peaks of curcumin and Eudragit E PO. Whereas, the spectrum of cur@EPO sample showed some significant changes. Peak at $3492 \mathrm{~cm}^{-1}$ in three curcumin polymer physical mixtures, as assigned to stretching of $\mathrm{OH}$ group in curcumin, disappeared in the spectrum of the cur@ EPO sample which suggested the involvement of the phenol $\mathrm{OH}$ group in the interaction (Gangwar et al., 2013). Fingerprint signals of curcumin in cur@EPO also changed significantly. Peak at $1628 \mathrm{~cm}^{-1}$ as assigned to the mixed stretching model of $\mathrm{C}=\mathrm{C}-\mathrm{C}=\mathrm{O}$ was shifted to $1625 \mathrm{~cm}^{-1}$ in cur@EPO SD. The microenvironment exist in different polymer may also exert impact on the wavenumber of $\mathrm{C}=\mathrm{C}-\mathrm{C}=\mathrm{O}$ in curcumin. Since curcumin could exist both in $\beta$-diketones and enolic form according to solution conditions. Band at $1603 \mathrm{~cm}^{-1}$ which was attributed to the symmetric aromatic ring stretching vibration shifted to $1576 \mathrm{~cm}^{-1}$ and become much broaden in the SD spectrum. Meanwhile, bands between $3000 \mathrm{~cm}^{-1}$ and $2800 \mathrm{~cm}^{-1}$ became weaker in the SD as compared with those of Eudragit E PO. Band at $2822 \mathrm{~cm}^{-1}$, assigned as $\mathrm{N}-\mathrm{CH}_{3}$ stretching, shifted to higher wavenumber at $2826 \mathrm{~cm}^{-1}$ in the cur@EPO SD (Pourreza, Golmohammadi, 2015). It is well known that in alkaline solution, curcumin tends to loss hydrogen and the color changes from yellow to red (Pourreza, Golmohammadi, 2015).Cur@EPO SD also shows characteristic red color of deprotonated curcumin. Along with FTIR result, it is anticipated that curcumin may donate one hydrogen to the dimethylaminoethyl methacrylate group of Eudragit E PO. Deprotonated curcumin may be stabilized by carbonyl groups of this polymer. When diluted in acetone, the color of cur@EPO reverted to yellow which was demonstrated in Figure S2. FTIR spectrum of cur@RLPO and cur@RSPO 
was similar with each other (Figure $4 \mathrm{~b}$ and $\mathrm{c}$ ). The $\mathrm{OH}$ stretching signal at about $3492 \mathrm{~cm}^{-1}$ was vanished in both of the SDs. But not like FTIR spectrum of cur@EPO, the signals between $3000 \mathrm{~cm}^{-1}$ and $2800 \mathrm{~cm}^{-1}$ in cur@RLPO and cur@RSPO resemble that of respective polymer. No signal reduction was observed in this wavenumber region. Nevertheless, band at $1140 \mathrm{~cm}^{-1}$ as attributed to ester C-O stretching shifted to higher wavenumber at $1142 \mathrm{~cm}^{-1}$ (Menjoge, Kulkarni, 2007). The results suggest that in cur@RLPO and cur@RSPO, curcumin may participate hydrogen bonding by $\mathrm{OH}$ group along with ester group in the polymer (Abbaspour, Sadeghi, Garekani, 2005).
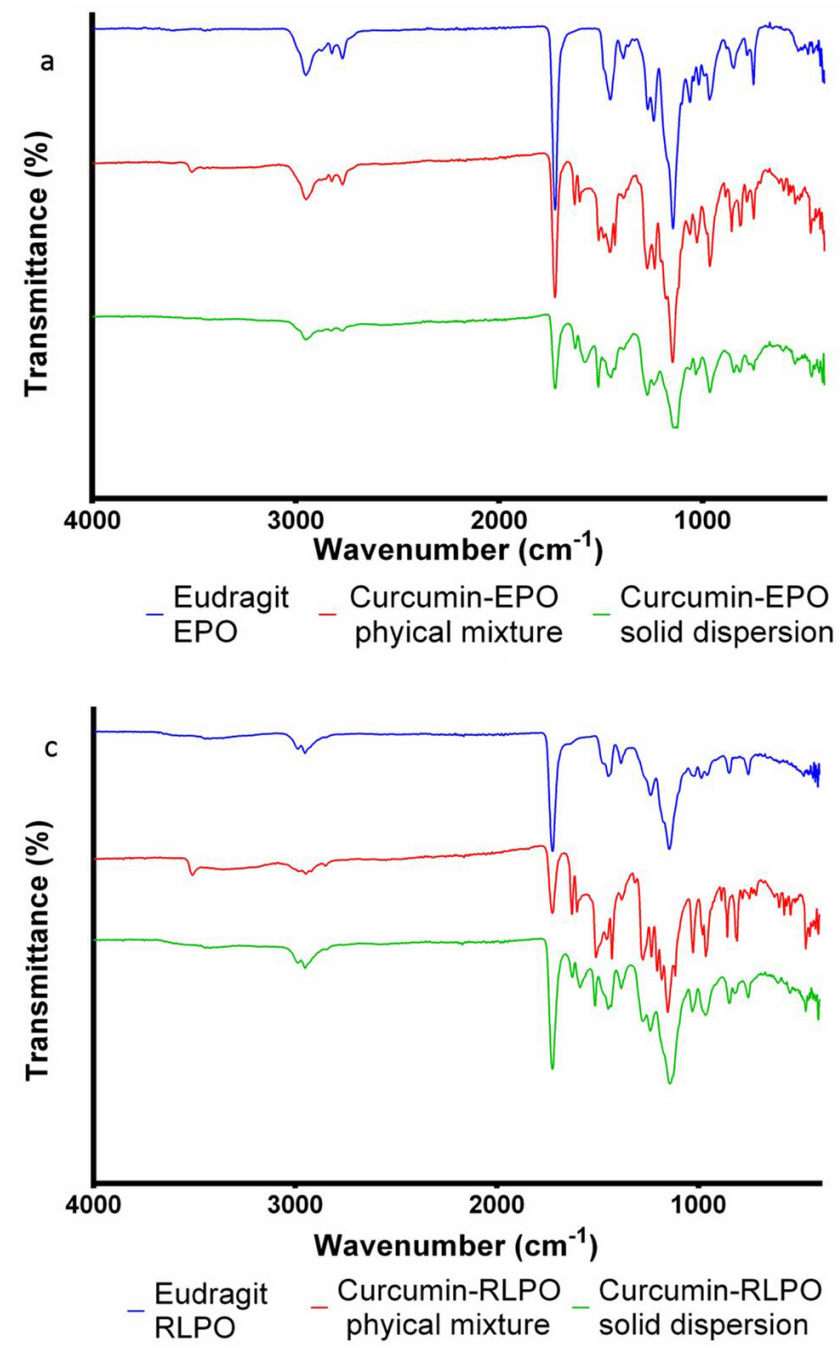

Both the color and FTIR results corroborate that when dispersed in polymer, curcumin may behave differently by losing one hydrogen in the Eudragit E PO matrix or forming hydrogen bond with surrounding Eudragit RS PO and Eudragit RL PO polymer. As we have mentioned, hydrogen bonding and ionic interaction are usually considered as the major attraction force between lipophilic drug and polymer in SD (Li et al., 2013; Ueda et al., 2015). But few studies gave detailed analysis and some results even contradicted with each other. Our results imply that polymer structure greatly impact on the way of interaction with loaded drug.
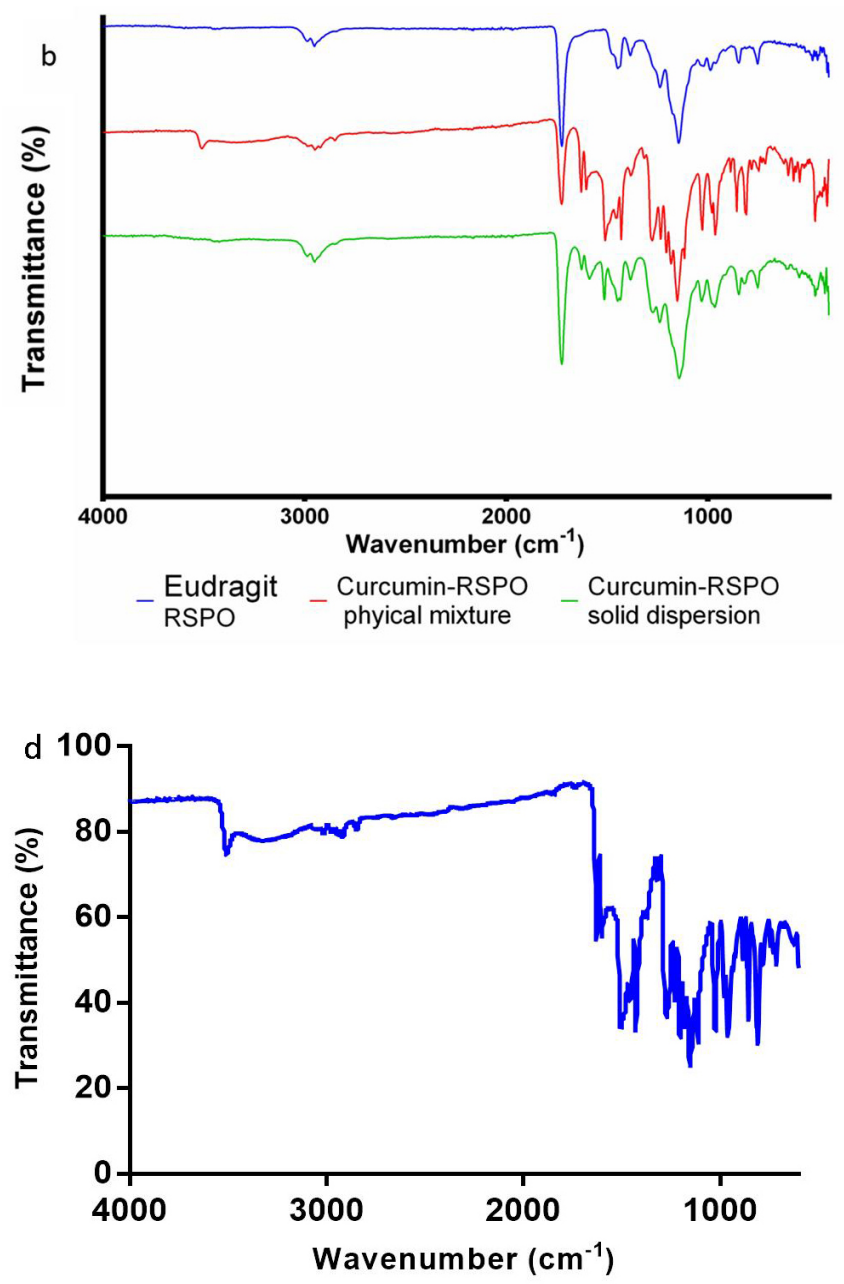

FIGURE 4 - Attenuated total reflecting-Fourier-transform infrared (ATR-FTIR) spectra of a: Eudragit E PO, physical mixture and curcumin Eudragit E PO solid dispersion; b: Eudragit RS PO, physical mixture and curcumin Eudragit RS PO solid dispersion; c: Eudragit RL PO, physical mixture and curcumin Eudragit RL PO solid dispersion; d: curcumin. 


\section{Release profile in simulated gastric fluid (SGF) and simulated intestinal fluid (SIF)}

Eudragit E PO is soluble in gastric fluid and become permeable in solution with $\mathrm{pH}$ higher than 5.0. On the other hand, both Eudragit RS PO and RL PO are not soluble in gastrointestinal fluid but have certain degree of swelling. The permeability of Eudragit RS PO is lower than that of Eudragit RL PO due to lower content of the quaternary ammonium group. Properties like solubility, swelling and permeability greatly influence the release and dissolution profile of entrapped drug. When added to SGF, cur@EPO released about 96\% of curcumin within 20 min which followed the typical burst release pattern (Figure 5). Longer incubation reduced the concentration of curcumin which may possibly due to rapid nucleation and crystallization as the consequence of oversaturation produced by the burst release (Sun, Lee, 2015). It is noteworthy to mention that Eudragit
EPO in the physical mixture, when dissolved in SGF, also facilitated the release of curcumin as proved by its higher release rate than that of pure curcumin and all other physical mixtures. Strong interaction between Eudragit EPO and curcumin may be accountable for the enhanced release. The dissolving promotion ability of Eudragit RS PO was also evident as higher level of curcumin was dissolved in the solution than its pristine form.Cur@RLPO released curcumin in a sustaining pattern which showed much higher level of release than that of cur@RSPO. Eudragit RL PO contains higher content of trimethylammonioethyl methacrylate that confer it higher permeability than Eudragit RS PO. As demonstrated in previous studies, higher permeability of Eudragit RL PO may be the main reason for the increase release of hydrophobic drugs (Apu et al., 2009; Kim et al., 2002). Release results in SGF indicate that curcumin release rate can be controlled by choice of polymer as well as formulation type (SD or physical mixture).

\section{Curcumin release in simulated gastric fluid}

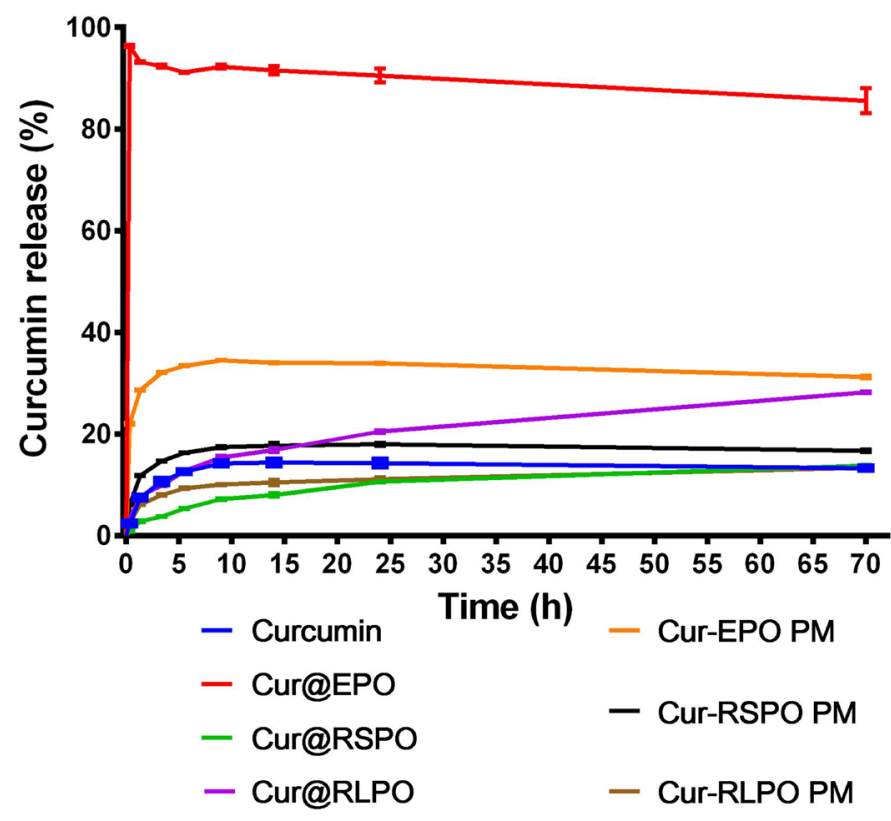

FIGURE 5 - Release profile in simulated gastric fluid (SGF) of curcumin, curcumin Eudragit E PO solid dispersion, curcumin Eudragit RS PO solid dispersion, curcumin Eudragit RL PO solid dispersion, curcumin and respective acrylic polymer including curcumin Eudragit E PO physical mixture (Cur-EPO PM), curcumin Eudragit RS PO physical mixture (Cur-RSPO PM) and curcumin Eudragit RL PO physical mixture (Cur-RLPO PM). Each text was repeated three times. 
The release patterns were markedly different in SIF (Figure 6). First of all, pure curcumin has higher solubility in SIF than that of SGF. About $30 \%$ of curcumin was dispersed in the SIF comparing with only $15 \%$ in SGF when incubated for $24 \mathrm{~h}$. Release speed of curcumin EPO physical mixture and curcumin RS PO physical mixture were close to that of pristine curcumin within $24 \mathrm{~h}$ of incubation. Longer of incubation helped to dissolve more curcumin in these two physical mixtures than pure curcumin. Physical mixture of RLPO and curcumin only released about $16 \%$ of curcumin within $24 \mathrm{~h}$. For the three SDs, sustaining release pattern was revealed.Cur@RLPO presented higher release speed than the other two groups. About $58 \%$ of curcumin in cur@RLPO was dissolved in SIF at the end of the test (70 h of incubation) comparing to 46\% in cur@EPO and 37\% in cur@RSPO. Since all the three polymers are not soluble in the SIF, curcumin can only release through diffusion. Higher permeability and swelling rate may increase the diffusion of curcumin from the matrix of polymer into the SIF. This was reinforced by the fact that in both SGF and SIF, cur@RLPO exhibited higher release level than that of cur@RSPO. Additionally, amorphous form of curcumin in SDs possesses higher molecular mobility and larger surface area, both of which contributing to their higher release extent (Li et al., 2013). During the release test, we observed that when added into SIF, the color of cur@RLPO and cur@RSPO changed from yellow to red, but the color of physical mixtures were not changed. Pristine curcumin could donate its first hydrogen (in the $\beta$-dicarbonyl group) in water at the $\mathrm{pH}$ around $7.8\left(\mathrm{pK}_{\mathrm{a} 1} \approx 7.8\right)$ (Heger et al., 2014). Our results imply that when existed in amorphous state, curcumin may loss its hydrogen at lower $\mathrm{pH}$ (SIF $\mathrm{pH}$ is 6.8).

\section{Curcumin release in simulated intestinal fluid}

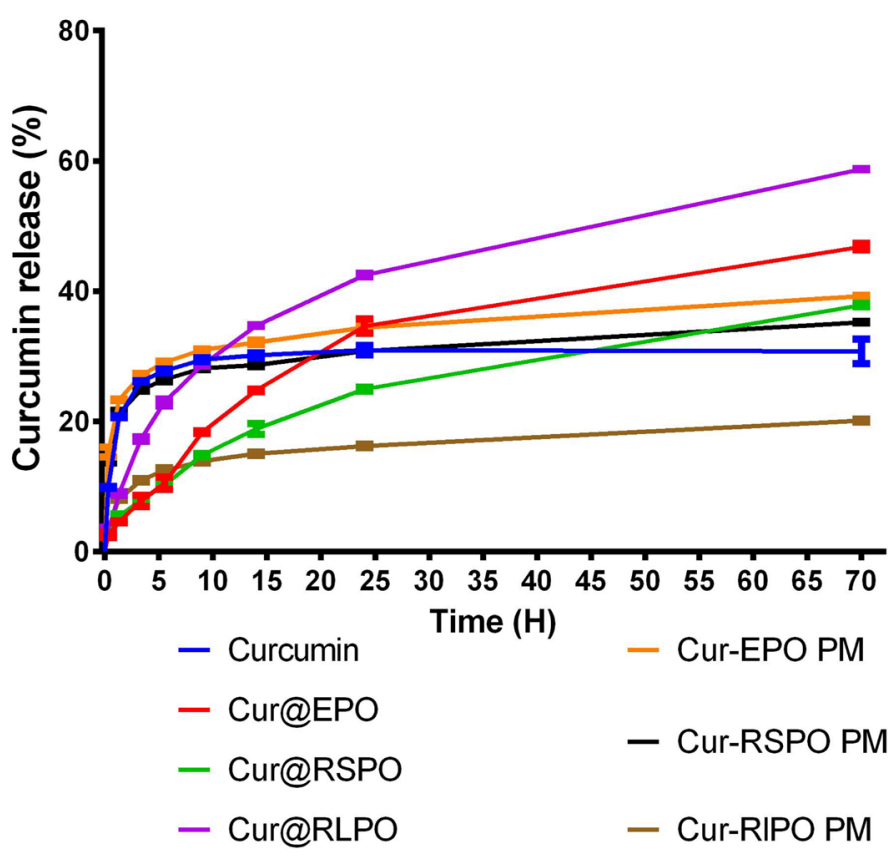

FIGURE 6 - Release profile in simulated intestinal fluid (SIF) of curcumin, curcumin Eudragit E PO solid dispersion, curcumin Eudragit RS PO solid dispersion, curcumin Eudragit RL PO solid dispersion, curcumin and respective acrylic polymer including curcumin Eudragit E PO physical mixture (Cur-EPO PM), curcumin Eudragit RS PO physical mixture (Cur-RSPO PM) and curcumin Eudragit RL PO physical mixture (Cur-RLPO PM). Each text was repeated three times. 
The release behaviors of curcumin from various formulations were evaluated by different kinetic release models which could provide better interpretation of the mechanisms controlling release. The most appropriate model was selected based on the values of correlation coefficient $\left(\mathrm{R}^{2}\right)$ as listed in Table II and III. It is evident that in SGF, except for cur@EPO, both cur@RSPO and cur@RLPO follow the Higuchi release rule which is usually employed to describe drug release complying with the Fick's law. The results suggest that for both cur@ RSPO and cur@RLPO, release of curcumin is mainly a diffusion controlled process (Kim et al., 2002). Also, cur@RLPO has larger release rate constant, k, than that of cur@RSPO, which is consistent with its faster release rate. All the physical mixtures along with pure curcumin presented Ritger-peppas release model with diffusional exponent (n) lower than 0.5 , indicating drug diffusion was the predominant release mechanism (Jiang et al., 2012). Release model for physical mixtures and pure curcumin in SIF also comply with Ritger-peppas release but with higher release rate constant than their respective values as revealed in Table II. Higher $\mathrm{pH}$ value is accountable for the faster release as aforementioned. However, when in SIF, all the SD samples follow the first order release model rather than Higuchi release as revealed in SGF. First order release is mainly used to describe drug release whose release rate is concentration dependent (Tonnesen, Masson, Loftsson, 2002). This phenomenon may be caused by the fact that curcumin has much higher solubility in SIF which contributes to its more freely diffusion from the polymer matrix to the release solution.

The release profiles as well as the release kinetics analysis imply that dissolution and release of curcumin may be modulated by both of varying polymer as well as formulation method. This is particular beneficial in pharmaceutic applications to fulfill the broad spectrum of therapeutic abilities of curcumin. It was observed that Cur RSPO PM could release curcumin more than Cur RSPO $\mathrm{SD}$. The release profile of curcumin is highly impacted by $\mathrm{pH}$ value. The PM was prepared by grinding with mortar which may break the crystal structure of curcumin and promote its release speed. On the other hand, curcumin in SD may have strong interaction with the polymer which retards its release into the solution. This may be responsible for the observed phenomena that Cur RSPO PM released curcumin more than Cur RSPO SD.

TABLE II - Release model and kinetics of curcumin, solid dispersion and physical mixture in simulated gastric fluid

\begin{tabular}{llcc}
\hline Sample group $^{\mathrm{a}}$ & Release model & Release equation $^{\mathrm{b}}$ & Release type \\
\hline Curcumin & Ritger-peppas & $\mathrm{Q}=33.97 * \mathrm{t} 0.1-27.75, \mathrm{R} 2=0.997$ & Fickian diffusion release \\
\hline Cur@EPO & - & - & Burst release \\
\hline Cur@RSPO & Higuchi rule & $\mathrm{Q}=2.51 * \mathrm{t} 1 / 2-0.44, \mathrm{R} 2=0.987$ & Diffusion controlled release \\
\hline Cur@RLPO & Higuchi rule & $\mathrm{Q}=4.78 * \mathrm{t} 1 / 2+1.39, \mathrm{R} 2=0.983$ & Diffusion controlled release \\
\hline Cur-EPO PM & Ritger-peppas & $\mathrm{Q}=36.01 * t 0.1-9.35, \mathrm{R} 2=0.967$ & Fickian diffusion release \\
\hline Cur-RSPO PM & Ritger-peppas & $\mathrm{Q}=32.51 * \mathrm{t} 0.1-22.37, \mathrm{R} 2=0.980$ & Fickian diffusion release \\
\hline Cur-RLPO PM & Ritger-peppas & $\mathrm{Q}=21.86 * t 0.1-16.74, \mathrm{R} 2=0.988$ & Fickian diffusion release
\end{tabular}

a Sample group include pure curcumin, curcumin Eudragit E PO solid dispersion (Cur@EPO), curcumin Eudragit RS PO solid dispersion (Cur@RSPO), curcumin Eudragit RL PO solid dispersion (Cur@RLPO), curcumin Eudragit E PO physical mixture (Cur-EPO PM), curcumin Eudragit RS PO physical mixture (Cur-RSPO PM), and curcumin Eudragit RL PO physical mixture (Cur-RLPO PM).

${ }^{b}$ Release equation was calculated based on each model equation, $\mathrm{Q}$ is release percentage at certain time point $\mathrm{t}$. 
TABLE III - Release model and kinetics of curcumin, solid dispersion and physical mixture in simulated intestinal fluid

\begin{tabular}{lccc}
\hline Sample group & Release model & Release equation & \\
\hline Curcumin & Ritger-peppas & $\mathrm{Q}=56.33 * \mathrm{t} 0.1-38.97, \mathrm{R} 2=0.956$ & Felease type \\
\hline Cur@EPO & Frist order & $\ln (1-\mathrm{Q})=-0.0172 * \mathrm{t}-0.0271, \mathrm{R} 2=0.991$ & Concentration controlled release \\
\hline cur@RSPO & Frist order & $\ln (1-\mathrm{Q})=-0.0108 * \mathrm{t}-0.0443, \mathrm{R} 2=0.975$ & Concentration controlled release \\
\hline Cur@RLPO & Frist order & $\ln (1-\mathrm{Q})=-0.0211 * \mathrm{t}-0.0976, \mathrm{R} 2=0.926$ & Concentration controlled release \\
\hline Cur-EPO PM & Ritger-peppas & $\mathrm{Q}=38.76 * \mathrm{t} 0.1-17.69, \mathrm{R} 2=0.968$ & Fickian diffusion release \\
\hline Cur-RSPO PM & Ritger-peppas & $\mathrm{Q}=34.37 * \mathrm{t} 0.1-15.22, \mathrm{R} 2=0.953$ & Fickian diffusion release \\
\hline Cur-RLPO PM & Ritger-peppas & $\mathrm{Q}=26.59 *$ t0.1-19.51, R2 $=0.985$ & Fickian diffusion release \\
\hline
\end{tabular}

aSample group include pure curcumin, curcumin Eudragit E PO solid dispersion (Cur@EPO), curcumin Eudragit RS PO solid dispersion (Cur@RSPO), curcumin Eudragit RL PO solid dispersion (Cur@RLPO), curcumin Eudragit E PO physical mixture (Cur-EPO PM), curcumin Eudragit RS PO physical mixture (Cur-RSPO PM), and curcumin Eudragit RL PO physical mixture (Cur-RLPO PM).

bRelease equation was calculated based on each model equation, $\mathrm{Q}$ is release percentage at certain time point $\mathrm{t}$.

\section{CONCLUSIONS}

In the present study, curcumin SDs formulated by three acrylic polymers were prepared by the solvent evaporation method. Their major physiochemical and control release profile were investigated. FTIR spectra results suggest that in cur@EPO, curcumin may transfer hydrogen to the dimethylaminoethyl methacrylate group of Eudragit EPO and thus showed deep red color. In contrast, hydrogen bonding may be responsible for the major interaction between curcumin and Eudragit RS PO/ Eudragit RL PO. Both DSC and XRD results confirm that in the three SDs, curcumin exists in amorphous state. Lower $\mathrm{pH}$ value in SGF stimulates the fast release of curcumin in cur@EPO while permeability influences the release in other two SDs. When in SIF, all the three SDs presented sustained release profile. Results in this study is helpful to shed light on how the structure of polymer impact on the major properties of curcumin contained SD which is promising to broaden the therapeutic applications of curcumin.

\section{ACKNOWLEDGEMENTS}

This research was supported by Fundamental Research Funds for the Central Universities
(JZ2018HGTB0244) and Anhui Natural Science Foundation (1808085QC66).

\section{CONFLICTS OF INTEREST}

No potential conflicts of interest were disclosed.

\section{REFERENCES}

Abbaspour MR, Sadeghi F, Garekani HA. Preparation and characterization of ibuprofen pellets based on Eudragit RS PO and RL PO or their combination. Int J Pharm. 2005;303(12):88-94.

Apu AS, Pathan AH, Shrestha D, Kibria G, Jalil RU. Investigation of In vitro Release Kinetics of Carbamazepine from Eudragit (R) RS PO and RL PO Matrix Tablets. Trop J Pharm Res. 2009;8(2):145-152.

Boyanapalli SSS, Huang Y, Su Z, Cheng D, Zhang C, Guo Y, et al. Pharmacokinetics and Pharmacodynamics of Curcumin in regulating anti-inflammatory and epigenetic gene expression. Biopharm Drug Dispos. 2018;39(6):289297.

Breitenbach J. Melt extrusion: from process to drug delivery technology. Eur J Pharm Biopharm. 2002;54(2):107-117.

Changdeo JS, Vinod M, Shankar KB, Rajaram CA. Physicochemical characterization and solubility 
enhancement studies of allopurinol solid dispersions. Braz $\mathbf{J}$ Pharm Sci. 2011;47(3):513-523.

Crotts G, Sheth A, Twist J, Ghebre-Sellassie I. Development of an enteric coating formulation and process for tablets primarily composed of a highly water-soluble, organic acid. Eur J Pharm Biopharm. 2001;51(1):71-76.

Deck LM, Hunsaker LA, Vander Jagt TA, Whalen LJ, Royer RE, Vander Jagt DL. Activation of anti-oxidant Nrf2 signaling by enone analogues of curcumin. Eur J Med Chem. 2018;143:854-865.

Desai S, Simonelli A, Higuchi W. Investigation of factors influencing release of solid drug dispersed in inert matrices. J Pharm Sci. 1965;54(10):1459-1464.

Esmaili M, Ghaffari SM, Moosavi-Movahedi Z, Atri MS, Sharifizadeh A, Farhadi M, et al. Beta casein-micelle as a nano vehicle for solubility enhancement of curcumin; food industry application. LWT-Food Sci Technol. 2011;44(10):2166-2172.

Gallardo D, Skalsky B, Kleinebudde P. Controlled release solid dosage forms using combinations of (meth)acrylate copolymers. Pharm Dev Technol. 2008;13(5):413-423.

Gangwar RK, Tomar GB, Dhumale VA, Zinjarde S, Sharma RB, Datar S. Curcumin conjugated silica nanoparticles for improving bioavailability and its anticancer applications. J Agr Food Chem. 2013;61(40):9632-9637.

Hasan M, Belhaj N, Benachour H, Barberi-Heyob M, Kahn CJF, Jabbari E, et al. Liposome encapsulation of curcumin: Physico-chemical characterizations and effects on MCF7 cancer cell proliferation. Int J Pharm. 2014;461(1-2):519-528.

Heger M, van Golen RF, Broekgaarden M, Michel MC. The molecular basis for the pharmacokinetics and pharmacodynamics of curcumin and its metabolites in relation to cancer. Pharmacol Rev. 2014;66(1):222-307.

Higuchi T. Mechanism of Sustained-Action Medication Theoretical analysis of rate of release of solid drugs dispersed in solid matrices. J Pharm Sci. 1963;52(12):1145-1149.

Jiang Y, Li FF, Luan YX, Cao WT, Ji XQ, Zhao LX et al. Formation of drug/surfactant catanionic vesicles and their application in sustained drug release. Int $\mathrm{J}$ Pharm. 2012;436(1-2):806-814.

Kakran M, Sahoo NG, Tan IL, Li L. Preparation of nanoparticles of poorly water-soluble antioxidant curcumin by antisolvent precipitation methods. J Nanopart Res. 2012;14(3):757-768.

Kharat M, Zhang G, McClements DJ. Stability of curcumin in oil-in-water emulsions: Impact of emulsifier type and concentration on chemical degradation. Food Res Int. 2018;111:178-186.

Kim BK, Hwang SJ, Park JB, Park HJ. Preparation and characterization of drug-loaded polymethacrylate microspheres by an emulsion solvent evaporation method. J Microencapsul. 2002;19(6):811-822.

Korsmeyer RW, Gurny R, Doelker E, Buri P, Peppas NA. Mechanisms of Solute Release from Porous Hydrophilic Polymers. Int J Pharm. 1983;15(1):25-35.

Li B, Konecke S, Wegiel LA, Taylor LS, Edgar KJ. Both solubility and chemical stability of curcumin are enhanced by solid dispersion in cellulose derivative matrices. Carbohyd Polym. 2013;98(1):1108-1116.

Li J, Lee IW, Shin GH, Chen X, Park HJ. CurcuminEudragit ${ }^{\circledR}$ E PO solid dispersion: A simple and potent method to solve the problems of curcumin. Eur J Pharm Biopharm. 2015a;94:322-332.

Li J, Shin GH, Chen X, Park HJ. Modified Curcumin with Hyaluronic Acid: Combination of Pro-drug and Nanomicelle Strategy to Address the Curcumin Challenge. Food Res Int. 2015b;69:202-208.

Liu HJ, Wang P, Zhang XY, Shen F, Gogos CG. Effects of extrusion process parameters on the dissolution behavior of indomethacin in Eudragit (R) E PO solid dispersions. Int J Pharm. 2010;383(1-2):161-169.

Menjoge AR, Kulkarni MG. Mechanistic investigations of phase behavior in Eudragit (R) blends. Int J Pharm. 2007;343(1-2):106-121.

Najib N, Suleiman MS. The Kinetics of Drug Release from Ethylcellulose Solid Dispersions. Drug Dev Ind Pharm. 1985;11(12):2169-2181.

Paradkar A, Ambike AA, Jadhav BK, Mahadik KR. Characterization of curcumin-PVP solid dispersion obtained by spray drying. Int J Pharm. 2004;271(1-2):281-286.

Paramera EI, Konteles SJ, Karathanos VT. Stability and release properties of curcumin encapsulated in Saccharomyces cerevisiae, beta-cyclodextrin and modified starch. Food Chem. 2011;125(3):913-922.

Pawar YB, Shete G, Popat D, Bansal AK. Phase behavior and oral bioavailability of amorphous Curcumin. Eur J Pharm Sci. 2012;47(1):56-64.

Pourreza N, Golmohammadi H. Application of curcumin nanoparticles in a lab-on-paper device as a simple and green pH probe. Talanta. 2015;131:136-141.

Sahoo J, Murthy PN, Biswal S, Ghosh M. Formulation of Sustained-Release Dosage Form of Verapamil Hydrochloride 
by Solid Dispersion Technique Using Eudragit RLPO or KollidonA (R) SR. Aaps Pharmscitech. 2009;10(1):27-33.

Sanmukhani J, Anovadiya A, Tripathi CB. Evaluation of antidepressant like activity of curcumin and its combination with fluoxetine and imipramine: an acute and chronic study. ACTA Pol Pharm. 2011;68:769-775.

Sanphui P, Goud NR, Khandavilli UB, Bhanoth S, Nangia A. New polymorphs of curcumin. Chem Commun. 2011;47: 5013-5015.

Soleimani H, Amini A, Taheri S, Sajadi E, Shafikhani S, Schuger LA, et al. The effect of combined photobiomodulation and curcumin on skin wound healing in type I diabetes in rats. J Photoch Photobio B. 2018;181:23-30.

Sun DD, Lee PI. Probing the mechanisms of drug release from amorphous solid dispersions in medium-soluble and mediuminsoluble carriers. J Control Release. 2015;211:85-93.

Tønnesen HH, Karlsen J. Studies on curcumin and curcuminoids. Zeitschrift für Lebensmittel-Untersuchung und Forschung. 1985;180(5):402-404.

Takka S, Rajbhandari S, Sakr A. Effect of anionic polymers on the release of propranolol hydrochloride from matrix tablets. Eur J Pharm Biopharm. 2001;52(1):75-82.

Tang B, Ma L, Wang HY, Zhang Y. Study on the supramolecular interaction of curcumin and $\beta$-cyclodextrin by spectrophotometry and its analytical application. J Agr Food Chem. 2002;50(6):1355-1361.

Thorat AA, Dalvi SV. Solid-state phase transformations and storage stability of curcumin polymorphs. Cryst Growth Des. 2015;15:1757-1770.

Tonnesen HH, Masson M, Loftsson T. Studies of curcumin and curcuminoids. XXVII. Cyclodextrin complexation: solubility, chemical and photochemical stability. Int J Pharm. 2002;244(1-2):127-135.

Ueda H, Wakabayashi S, Kikuchi J, Ida Y, Kadota K, Tozuka Y. Anomalous role change of tertiary amino and ester groups as hydrogen acceptors in eudragit e based solid dispersion depending on the concentration of Naproxen. Mol Pharmaceutics. 2015;12(4):1050-1061.

Varshosaz J, Faghihian H, Rastgoo K. Preparation and characterization of metoprolol controlled-release solid dispersions. Drug Deliv. 2006;13(4):295-302.

Wegiel LA, Zhao YH, Mauer LJ, Edgar KJ, Taylor LS. Curcumin amorphous solid dispersions: the influence of intra and intermolecular bonding on physical stability. Pharm Dev Technol. 2014;19(8):976-986.

$\mathrm{Wu} \mathrm{CB}$, McGinity JW. Influence of methylparaben as a solid-state plasticizer on the physicochemical properties of Eudragit (R) RS PO hot-melt extrudates. Eur J Pharm Biopharm. 2003;56(1):95-100.

Zhang F, Koh GY, Jeansonne DP, Hollingsworth J, Russo PS, Vicente G, et al. A novel solubility-enhanced curcumin formulation showing stability and maintenance of anticancer activity. J Pharm Sci. 2011;100(7):2778-2789.

Received for publication on $08^{\text {th }}$ November 2019 Accepted for publication on $31^{\text {st }}$ March 2020 